\title{
Effects of linagliptin vs glimepiride on cognitive performance in type 2 diabetes: results of the randomised double-blind, active-controlled CAROLINA-COGNITION study
}

\author{
Geert Jan Biessels ${ }^{1} \cdot$ Chloë Verhagen $^{1} \cdot$ Jolien Janssen $^{1} \cdot$ Esther van den Berg ${ }^{1,2} \cdot$ Gudrun Wallenstein $^{3}$. \\ Bernard Zinman ${ }^{4} \cdot$ Mark A. Espeland $^{5}$. Odd Erik Johansen ${ }^{6}$ (I)
}

Received: 7 August 2020 / Accepted: 11 December 2020 / Published online: 9 February 2021

(C) The Author(s) 2021

\begin{abstract}
Aims/hypothesis Type 2 diabetes, particularly with concomitant CVD, is associated with an increased risk of cognitive impairment. We assessed the effect on accelerated cognitive decline (ACD) of the DPP-4 inhibitor linagliptin vs the sulfonylurea glimepiride in individuals with type 2 diabetes.

Methods The CAROLINA-COGNITION study was part of the randomised, double-blind, active-controlled CAROLINA trial that evaluated the cardiovascular safety of linagliptin vs glimepiride in individuals with age $\geq 40$ and $\leq 85$ years and $\mathrm{HbA}_{1 \mathrm{c}} 48$ $69 \mathrm{mmol} / \mathrm{mol}(6.5-8.5 \%)$ receiving standard care, excluding insulin therapy. Participants were randomised 1:1 using an interactive telephone- and web-based system and treatment assignment was determined by a computer-generated random sequence with stratification by center. The primary cognitive outcome was occurrence of ACD at end of follow-up, defined as a regressionbased index score $\leq 16$ th percentile on either the Mini-Mental State Examination (MMSE) or a composite measure of attention and executive functioning, in participants with a baseline MMSE score $\geq 24$. Prespecified additional analyses included effects on ACD at week 160, in subgroups (sex, age, race, ethnicity, depressive symptoms, cardiovascular risk, duration of type 2 diabetes, albuminuria), and absolute changes in cognitive performance. Participants, caregivers, and people involved in measurements, examinations or adjudication, were all masked to treatment assignment.

Results Of 6033 participants recruited from hospital and primary care sites, 3163 (38.0\% female, mean age/diabetes duration 64/ 7.6 years, MMSE score 28.5, $\mathrm{HbA}_{1 \mathrm{c}} 54 \mathrm{mmol} / \mathrm{mol}$ [7.1\%]) represent the CAROLINA-COGNITION cohort. Over median 6.1 years, ACD occurred in $27.8 \%$ (449/1618, linagliptin) vs $27.6 \%$ (426/1545, glimepiride), OR 1.01 (95\% CI 0.86, 1.18). Also, no differences in ACD were observed at week 160 (OR 1.07 [0.91, 1.25]), between treatments across subgroups, or for absolute cognitive changes.

Conclusions/interpretation In a large, international outcome trial in people with relatively early type 2 diabetes at elevated cardiovascular risk, no difference in risk for ACD was observed between linagliptin and glimepiride over 6.1 years.

Funding This study was sponsored by Boehringer Ingelheim.

Trial registration ClinicalTrials.gov NCT01243424.
\end{abstract}

Keywords Cardiovascular disease $\cdot$ Cognitive decline $\cdot$ DPP-4 inhibitors $\cdot$ Sulfonylureas $\cdot$ Type 2 diabetes

Geert Jan Biessels

G.J.Biessels@umcutrecht.nl

Odd Erik Johansen

odd_erikj@hotmail.com

1 Department of Neurology, UMC Utrecht Brain Center, University Medical Center Utrecht, Utrecht, the Netherlands

2 Department of Neurology, Erasmus MC - University Medical Center, Rotterdam, the Netherlands
3 Biostatistics and Data Sciences, Boehringer Ingelheim, Ingelheim, Germany

4 Lunenfeld-Tanenbaum Research Institute, Mount Sinai Hospital, University of Toronto, Toronto, Canada

5 Department of Biostatistics and Data Science, Wake Forest School of Medicine, Winston-Salem, NC, USA

6 Therapeutic Area Cardiometabolism, Boehringer Ingelheim, Asker, Norway 


\section{Research in context}

\section{What is already known about this subject?}

- $\quad$ Type 2 diabetes is associated with increased risk for cognitive impairment, but there are few RCTs that have assessed whether glucose-lowering therapies might modulate this risk

- The CAROLINA trial, an RCT that included 6033 participants with type 2 diabetes at elevated cardiovascular risk, found no difference in cardiovascular risk between linagliptin and glimepiride, despite a differential effect on risk for hypoglycaemia and weight gain

What is the key question?

- Are there differing effects of linagliptin and glimepiride on accelerated cognitive decline (ACD) in participants of CAROLINA-COGNITION?

What are the new findings?

- Amongst the 3163 participants, followed for a median period of 6.1 years, the risk for cognitive decline was higher with advancing age and in those with established CVD, but there was no difference in risk for ACD between those treated with linagliptin or glimepiride

\section{How might this impact on clinical practice in the foreseeable future?}

- These findings do not support previous hypotheses that DPP-4 inhibitors or sulfonylureas may modulate cognitive function positively; however, the neutral effect on cognitive function also provides reassurance that these medications do not negatively affect cognition

- Preventing cognitive impairment remains an unmet need in type 2 diabetes

$\begin{array}{ll}\text { Abbreviations } \\ \text { ACD } & \text { Accelerated cognitive decline } \\ \text { AE } & \text { Adverse event } \\ \text { CES-D } & \text { Center for Epidemiologic Studies Depression Scale } \\ \text { DPP-4 } & \text { Dipeptidyl peptidase-4 } \\ \text { GLP-1 } & \text { Glucagon-like peptide-1 } \\ \text { MCI } & \text { Mild cognitive impairment } \\ \text { MMSE } & \text { Mini-Mental State Examination } \\ \text { RBI } & \text { Regression-based index } \\ \text { TMT } & \text { Trail making test } \\ \text { UACR } & \text { Urine albumin/creatinine ratio } \\ \text { VFT } & \text { Verbal fluency test }\end{array}$

\section{Introduction}

Type 2 diabetes is associated with increased risk for cognitive impairment, both mild cognitive impairment (MCI) and dementia [1], in particular in those patients with coexisting CVD [2]. More subtle cognitive changes, also referred to as cognitive decrements, can be encountered in patients with type 2 diabetes of all age groups, usually with only modest deterioration over time $[1,3]$. Yet, accelerated cognitive decline (ACD), which may evolve to MCI or dementia, predominantly occurs in higher numbers in the older age groups [1,3-5]. This is worrying, since in people 60 years or older with type 2 diabetes, the annual risk of dementia has been observed at 2.6\% [6], and older people will constitute the majority of individuals with type 2 diabetes in the USA and most other developed countries in the next decades, with type 2 diabetes currently estimated to affect $19.3 \%$ of people 65 years or older worldwide [7]. Cognitive dysfunction in type 2 diabetes, in particular severe forms, is an independent predictor for adverse clinical outcomes such as hypoglycaemia, cardiovascular events and death [1, 8].

The pathophysiology of cognitive decline in type 2 diabetes is complex, likely sharing pathways with conditions such as Alzheimer's disease and vascular dementia [1, 9, 10]. The hallmark of type 2 diabetes, hyperglycaemia, has also been associated with incident dementia, both in people with and without type 2 diabetes [11], in the latter including a possible nonlinearity where both high and low $\mathrm{HbA}_{1 \mathrm{c}}$ levels are linked to poorer cognitive functioning [12]. However, interventions aimed at achieving near-normoglycaemia have not been shown to reduce the risk for cognitive decline [13]. Given the association between type 2 diabetes and MCI and dementia, it is therefore of interest to assess whether the non-glycaemic effects of specific glucose-lowering medications affect cognitive function. This is also of particular relevance in light of some conflicting results on cognitive functioning reported with the use of metformin $[5,14,15]$ and insulin $[16,17]$.

Incretin-based glucose-lowering medications have emerged as a potential therapeutic agent for Alzheimer's disease [18]. The underlying hypothesis of potential benefit is related to the nonglycaemic effects of this class of drug, involving a variety of direct or indirect targets in the brain for glucagon-like peptide-1 (GLP-1) receptor agonists and dipeptidyl peptidase (DPP)-4 
inhibitors, including neuronal cells, different glial cells and neuronal precursor cells [19]. Animal data show that DPP-4 inhibitors may suppress blood-brain barrier disruption and attenuate cerebral oxidative stress or neuroinflammation, and may reduce brain damage following a stroke [19]. The findings of a recent, adequately powered, clinical study comparing effects on cognitive function of dulaglutide vs placebo also support this notion: a modest beneficial effect on a cognitive outcome, defined as first occurrence of a follow-up score on the Montreal Cognitive Assessment or Digit Symbol Substitution Test that was 1.5 SD or more below the baseline mean score in the participant's country, was seen in an exploratory analysis [20].

Another widely used class of glucose-lowering medication investigated for effects on the cerebrovascular system are sulfonylureas, which also have been suggested to have potential neuron protective effects in various neurological disorders $[21,22]$. However, its association with increased risk of hypoglycaemia could offset such benefits, as hypoglycaemia may increase the risk of cognitive impairment in type 2 diabetes $[23,24]$. Preclinical studies suggest that sulfonylureas reduce brain infarct volumes and oedema and haemorrhagic conversion, and improve outcomes in rodent models of ischaemic stroke, mainly related to involvement of the sulfonylurea receptor 1 [22]. To date, however, there are no robust clinical data to support a benefit of either DPP-4 inhibitors or sulfonylureas on cognitive functioning [5, 13, 25, 26], (see also literature review conducted up to 10 July 2020, electronic supplementary material [ESM] Table 1), and no direct comparisons have been performed.

The CARdiovascular Outcome study of LINAgliptin versus glimepiride in type 2 diabetes (CAROLINA) was the first head-to-head active-controlled outcome trial of these two classes of medication, and involved 6033 participants with relatively early type 2 diabetes with cardiovascular safety as primary outcome. It demonstrated no relative benefits of linagliptin vs glimepiride, when given in addition to the usual standard of care, for the primary composite outcome of cardiovascular death, non-fatal myocardial infarction and non-fatal stroke (HR 0.98 [95\% CI 0.84, 1.13]), and fatal/non-fatal stroke $(0.86[0.66,1.12])$ [27]. CAROLINA also implemented a cognition substudy to assess relative effects on global, and domain-specific, cognitive function [28], and herein we report the impact on cognitive decline and on global cognitive function using a validated standardised cognitive test battery sensitive to relatively mild cognitive changes.

\section{Methods}

\section{Trial design and participants}

The CAROLINA-COGNITION study was an integral part of the CAROLINA trial, a randomised, active comparator, double-blind study to evaluate the cardiovascular safety of linagliptin $5 \mathrm{mg}$ vs glimepiride $1-4 \mathrm{mg}$, and was event driven until a minimum of 631 patients had experienced the primary outcome event. Over 600 centres across 43 countries participated, enrolling adults with relatively early type 2 diabetes $\left(\mathrm{HbA}_{1 \mathrm{c}} 48-69 \mathrm{mmol} / \mathrm{mol}\right.$ [6.5-8.5\%]) at elevated risk of cardiovascular events receiving usual care while excluding those already on insulin therapy [27]. Elevated cardiovascular risk was defined as: previous vascular disease and/or evidence of vascular-related end-organ damage and/or age $\geq 70 \mathrm{and} /$ or a minimum of two predefined cardiovascular risk factors. Detailed inclusion and exclusion criteria have previously been published [27] and are available in the supplementary material (ESM Table 2).

Glimepiride was initiated at $1 \mathrm{mg}$ and up-titrated every 4 weeks during the first 16 weeks to a maximal dose of $4 \mathrm{mg}$. Investigators were encouraged to use glucoselowering rescue medication if glycaemic control was insufficient and to manage all other cardiovascular risk factors according to applicable guidelines and current standards of care.

\section{CAROLINA-COGNITION study}

The cognition substudy aimed to investigate if treatment with linagliptin compared with glimepiride, once daily on top of standard care, could prevent ACD [28]. The study protocol was approved by the institutional review board or independent ethics committee from each site, and all participants provided written informed consent. Because of the cognitive test procedures, participants were required to live in a country that had a native language built on the Latin alphabet. Participants were included in the primary analysis if: (1) their years of formal education were captured; (2) they had a valid baseline cognitive assessment; and (3) they had a valid follow-up cognitive assessment within 7 days after their last study-drug intake. Those with Mini-Mental State Examination (MMSE) score $<24$ at baseline were excluded, since the cognition substudy intended to investigate if treatment with linagliptin could prevent occurrence of cognitive decline.

\section{Procedures}

A comprehensive description of the cognitive assessment and corresponding procedures has previously been published [28]. In brief, to measure ACD, two cognitive measures were used: (1) the MMSE, a widely used and validated screening test for global cognition in older adults [29], for which a score below 24 is generally accepted to indicate cognitive impairment [30]; and (2) a more sensitive, domain-specific composite measure of attention and executive functioning combining performance on both Verbal Fluency Test (VFT) and Trail 
Making Test (TMT) [28, 31, 32]. For the VFT, F - A - S were used for letter fluency, and animals for category.

As depression is a known confounder of cognitive performance, participants were also asked to complete the Center for Epidemiologic Studies Depression Scale (CES-D), a 20-item questionnaire on depressive symptoms experienced over the last week, during each visit [33]. A score $\geq 16$ indicates presence of depressive symptoms.

Further details around the MMSE, attention and executive functioning score and CES-D have been published previously [28]. Additional details on the attention and executive functioning derivation are also described in ESM Methods. All tests were administered by trained investigators, or designated site personnel.

\section{Time-windows}

Cognitive assessments were conducted at baseline, after 160 weeks of follow-up and at end of follow-up provided that an MMSE score $\geq 24$ was obtained at baseline. End of followup occurred either when a participant discontinued study medication/discontinued trial or at the end of the main trial (see ESM Fig. 1 for description of handling of missing cognitive assessments).

\section{Cognitive outcomes}

Primary cognitive outcome The primary cognitive outcome was defined as the incidence of ACD at end of follow-up, using a regression-based index (RBI). The RBI score reflects the difference between the observed and predicted cognitive score for each individual, and takes potential confounders (i.e. baseline test performance, age, years of formal education, sex, race and test-retest interval) into account at subject level, as opposed to raw change in test scores. The derivation and calculation of the $\mathrm{RBI}$ is previously described [28] and additional information on the calculation of the predicted score can be found in the supplementary material (ESM Methods). Participants were classified as having ACD when their cognitive decline score was at or below the 16th percentile of the RBI score of the total substudy population: this cut-off was chosen as it corresponds approximately to one SD below the mean. Participants with a valid baseline cognitive assessment who did not understand the cognitive test instructions at follow-up were also classified as having ACD. The ACD classification thus identifies individuals that decline faster than would be expected compared with other participants, while considering the confounders listed above at an individual level. Considerations for handling potential protocol deviations and missing data points are described in the supplementary material (ESM Table 3). A secondary analysis, to check the robustness of the results, was also performed, using the 10th percentile as cut-off (instead of the 16th percentile) to define ACD. Predefined sensitivity analyses, post hoc analyses, handling of potential protocol deviations and missing data are described in ESM Table 3, ESM Fig. 1 and ESM Fig. 2.

Subgroup analyses Subgroup analyses similar to the primary analysis of the primary cognitive outcome were predefined for the following baseline variables: sex (male/female), age ( $<70$, $\geq 70$ years), race (black, white, Asian, other), ethnicity (Latino/ Hispanic, non-Latino/Hispanic), CES-D score $(<16, \geq 16$ and median split), duration of type 2 diabetes ( $\leq 1$ year, $>1$ to $\leq 5$ years, $>5$ to $\leq 10$ years, $>10$ years), urine albumin/ creatinine ratio (UACR) $(<30 \mathrm{mg} / \mathrm{mmol}, \geq 30 \mathrm{mg} / \mathrm{mmol}$ to $\leq 300 \mathrm{mg} / \mathrm{mmol},>300 \mathrm{mg} / \mathrm{mmol})$, and cardiovascular risk categories (high, moderate-high, moderate-low or low).

A post hoc subgroup analysis for the primary cognitive outcome was also conducted for participants with age $<75$ vs $\geq 75$ years.

Secondary cognitive endpoints Secondary cognitive endpoints include: (1) the incidence of ACD at end of follow-up, using the 10th percentile; (2) the incidence of ACD after 160 weeks of follow-up, both with the 16th and the 10th percentile; and (3) the incidence of ACD using the 16 th percentile of the $\mathrm{z}$ score of the cognitive measures (i.e. MMSE and attention and executive function) instead of RBI score, both at end of follow-up and after 160 weeks.

Further cognitive endpoints Further predefined endpoints included: (1) the incidence of ACD defined as an MMSE score $<24$ or a decline of $>4$ points on MMSE, relative to baseline; (2) incidence of depression defined as a CES-D score of $\geq 16$; and (3) changes from baseline for all cognitive (sub)tests of VFT, TMT and MMSE. All endpoints were derived after 160 weeks of follow-up and at end of followup for both treatment groups.

\section{Post hoc assessment of metabolic response and hypoglycaemia}

Change from baseline for $\mathrm{HbA}_{1 \mathrm{c}}$ and weight was analysed, as was the occurrence of hypoglycaemia. The latter was not adjudicated, but reported by investigators based on participant symptoms or laboratory values according to: (1) symptomatic hypoglycaemic adverse event (AE) with plasma glucose $\leq 3.9 \mathrm{mmol} / \mathrm{l}$, or hypoglycaemic AE with plasma glucose $<3.0 \mathrm{mmol} / \mathrm{l}$; (2) severe hypoglycaemic AE defined as requiring the assistance of another person to actively administer carbohydrate, glucagon or other resuscitative actions; or (3) hypoglycaemic $\mathrm{AE}$ leading to hospitalisation.

\section{Statistical analyses}

For the analysis of the primary and secondary endpoints, the incidence of ACD at end of follow-up was compared between 
treatment groups using a logistic regression model including treatment as a factor. The OR, along with the $95 \%$ Wald CI and two-sided $p$ value, were calculated for treatment comparison. For subgroup analyses, terms for treatment, subgroup, and subgroup-by-treatment interactions were included in the logistic regression model.

The continuous cognition endpoints, change from baseline, were analysed by a linear mixed-effects model for repeated measures. The restricted maximum likelihood estimation method was involved, and the Kenward-Roger method was used to adjust standard errors and estimate denominator $d f$. For the pairwise comparisons of linagliptin vs glimepiride, the differences between the expected means were estimated by the difference in the corresponding adjusted means. Twosided $95 \%$ CIs based on the $t$ distribution were also computed. Data after 160 weeks of follow-up and at end of follow-up were included in these analyses. In all analyses, participants were grouped by original random treatment assignment.

The repeated-measures analysis described above was also applied to change from baseline over time in $\mathrm{HbA}_{1 \mathrm{c}}$ and weight. Data were included up to the planned week that could theoretically be achieved by all participants.

Hypoglycaemic events that occurred during treatment, or within 7 days after the last dose of a study drug, were analysed as time-to-first-event using a Cox proportional hazards model, with treatment as a factor (two-sided $p$ value from Wald's $\chi^{2}$ test).

All analyses were conducted with SAS version 9.4 (SAS Institute, Cary, NC, USA).

\section{Sample size considerations}

For the primary outcome it was expected that approximately 20-22\% (ESM Methods) of the whole cohort would meet the criteria for ACD [23]. Assuming at least 4500 participants at baseline, with cognitive follow-up, this would allow the detection of an RR reduction of $20 \%$ in the linagliptin group relative to the glimepiride group with a power of $80 \%$ and twosided $\alpha$ of 0.05 .

\section{Results}

Out of 4529 participants from countries eligible for the CAROLINA-COGNITION study, 4018 were included at baseline. Reasons for exclusion at baseline were missing information on education level or language or illiteracy $(n=$ 232), missing MMSE or MMSE <24 ( $n=279)$. In total 3163 out of 4018 participants from 31 countries (ESM Fig. 2 and ESM Fig. 3) had at least one valid follow-up cognitive assessment and were included in the primary analysis. Reasons for follow-up assessment being missing, or excluded (following predefined criteria) [23], were: no valid follow-up assessment of cognition at any visit ( $n=566$, of whom $n=161$ died before their scheduled assessment); and the only available cognitive assessment being more than 7 days after their last medication intake $(n=289)$.

Baseline clinical characteristics and cognitive scores were well balanced between the two treatment arms (Table 1). Overall, mean \pm SD age was $64.4 \pm 9.2$ years $(14.2 \% \geq$ 75 years), mean duration of type 2 diabetes $7.6 \pm 6.1$ years, and mean years of formal education 10.8 \pm 3.5 . Mean BMI was $30.8 \pm 5.0 \mathrm{~kg} / \mathrm{m}^{2}$ and $\mathrm{HbA}_{1 \mathrm{c}} 54 \pm 6.1 \mathrm{mmol} / \mathrm{mol}(7.1 \pm 0.6 \%)$. Mean MMSE at baseline was $28.5 \pm 1.7$ and CES-D $9.0 \pm 8.2$ with $16.7 \%$ scoring CES-D $\geq 16$.

Median ( $\min -\max )$ time between randomisation and assessment at end of follow-up was $6.12(0.02-7.42)$ years, with no significant differences between treatment arms. Baseline characteristics of participants who dropped out of the substudy without having a post-baseline assessment were balanced between treatment groups (ESM Table 4) and their profile differed only modestly from the overall population with slightly more prevalent cardiovascular disease at baseline and slightly higher proportion reporting depressive symptoms.

\section{Primary cognitive outcome}

Of the 3163 participants that were included in the primary analysis, 449/1618 (27.8\%) in the linagliptin group and 426/ $1545(27.6 \%)$ in the glimepiride group had ACD during follow-up. In those with ACD, the MMSE changed by -2.5 \pm 3.6 points and the attention and executive function $\mathrm{z}$ score by $-0.7 \pm 1.0$ points, as opposed to $0.4 \pm 1.6$ and $0.1 \pm 0.7$ in those without ACD (ESM Table 5). There was no difference in occurrence of ACD between treatment arms (1.01 [95\% CI $0.86,1.18]$ ), nor in the subgroup analyses (Fig. 1a), although in both groups, the proportion with ACD was higher in those with higher age, in women, in those with Latino/Hispanic ethnicity, in those with vascular disease, and in those with higher CES-D scores at baseline. In a post hoc defined analysis amongst those $\geq 75$ years of age, ACD occurred in 82/226 $(36.3 \%)$ in the linagliptin group and 67/224 (29.9\%) in the glimepiride group $(1.33$ [0.90, 1.98]).

Sensitivity analyses supported the results of the primary analysis (Fig. 1b, ESM Table 6).

\section{Secondary and further cognitive outcomes}

The analysis of ACD after 160 weeks of follow-up showed consistent results with the primary analysis (linagliptin: 446/ 1618 [27.6\%], glimepiride: 406/1545 [26.3\%], 1.07 [95\% CI $0.91,1.25])$. Similar results were found with the secondary analysis applying the 10th percentile as cut-off for the RBI score at end of follow-up (linagliptin 296/1618 [18.3\%], glimepiride 291/1545 [18.8\%], 0.96 [0.81, 1.15]), (ESM 
Table 1 Baseline characteristics by treatment group

\begin{tabular}{|c|c|c|}
\hline Variables & $\begin{array}{l}\text { Linagliptin } \\
(n=1618)\end{array}$ & $\begin{array}{l}\text { Glimepiride } \\
(n=1545)\end{array}$ \\
\hline Male/female & $1002(61.9) / 616(38.1)$ & $958(62.0) / 587(38.0)$ \\
\hline Age, years & $64.4 \pm 9.1$ & $64.4 \pm 9.3$ \\
\hline \multicolumn{3}{|l|}{ Medical history } \\
\hline History of myocardial infarction & $226(14.0)$ & $187(12.1)$ \\
\hline History of cerebrovascular disease & $169(10.4)$ & $154(10.0)$ \\
\hline Atrial fibrillation & $86(5.3)$ & $74(4.8)$ \\
\hline Known coronary artery disease & $394(24.4)$ & $366(23.7)$ \\
\hline Education level, years & $10.8 \pm 3.4$ & $10.8 \pm 3.5$ \\
\hline BMI, $\mathrm{kg} / \mathrm{m}^{2}$ & $30.8 \pm 5.0$ & $30.7 \pm 4.9$ \\
\hline MMSE score & $28.5 \pm 1.7$ & $28.5 \pm 1.7$ \\
\hline Depression score according to CES-D & $8.7 \pm 8.0$ & $9.3 \pm 8.3$ \\
\hline$<16$ & $1335(82.5)$ & $1242(80.4)$ \\
\hline$\geq 16$ & $250(15.5)$ & $278(18.0)$ \\
\hline Missing & $33(2.0)$ & $25(1.6)$ \\
\hline eGFR (MDRD), $\mathrm{ml} \mathrm{min}^{-1}[1.73 \mathrm{~m}]^{-2}$ & $75.8 \pm 19.0$ & $76.9 \pm 18.8$ \\
\hline Type 2 diabetes duration, years & $7.7 \pm 6.2$ & $7.4 \pm 5.9$ \\
\hline $\mathrm{HbA}_{1 \mathrm{c}}, \mathrm{mmol} / \mathrm{mol}(\%)$ & $54.3 \pm 6.0(7.1 \pm 0.5)$ & $54.5 \pm 6.2(7.1 \pm 0.6)$ \\
\hline Fasting plasma glucose, mmol/1 & $7.8 \pm 1.7$ & $7.8 \pm 1.6$ \\
\hline \multicolumn{3}{|l|}{ Glucose-lowering medications } \\
\hline Metformin & $1348(83.3)$ & $1306(84.5)$ \\
\hline Sulfonylurea & $434(26.8)$ & $422(27.3)$ \\
\hline Glinide & $13(0.8)$ & $13(0.8)$ \\
\hline$\alpha$-glucosidase inhibitor & $43(2.7)$ & $34(2.2)$ \\
\hline Thiazolidinedione & $1(0.1)$ & $2(0.1)$ \\
\hline \multicolumn{3}{|l|}{ Cardiovascular medications } \\
\hline Lipid-lowering & $1197(74.0)$ & $1180(76.4)$ \\
\hline Statins & $1111(68.7)$ & $1113(72.0)$ \\
\hline Antihypertensives & $1428(88.3)$ & $1387(89.8)$ \\
\hline Systolic BP, mmHg & $135.9 \pm 15.9$ & $136.2 \pm 16.4$ \\
\hline Diastolic BP, mmHg & $78.8 \pm 9.5$ & $78.8 \pm 9.3$ \\
\hline LDL-cholesterol, mmol/1 & $2.4 \pm 0.9$ & $2.4 \pm 0.9$ \\
\hline
\end{tabular}

Data are $n(\%)$ or mean \pm SD unless otherwise stated

MDRD, Modification of Diet in Renal Disease study equation
Tables 6-8). Also, no differences were found between the treatment arms in the subgroups (Fig. 1a), or in a sensitivity analysis including 3452 participants (linagliptin: 1766, glimepiride: 1686), regardless of whether their latest postbaseline assessment was conducted later than 7 days after treatment discontinuation (1.04 [0.89, 1.21]) (ESM Fig. 2).

Absolute changes from baseline in MMSE, VFT and TMT scores were similar between treatment groups, both after 160 weeks and at end of follow-up (Table 2).

The proportion of participants experiencing substantial depressive symptoms (CES-D $\geq 16$ ) after 160 weeks of follow-up was lower for those who received linagliptin (22.9\%) compared with glimepiride (26.1\%), 0.84 (0.71,
1.00). However, this difference was attenuated at the end of follow-up (linagliptin: $21.3 \%$, glimepiride: $23.8 \%, 0.86$ [0.71, $1.05])$.

\section{Effects on metabolic variables and hypoglycaemia}

The mean $( \pm \mathrm{SD})$ dose of glimepiride over the trial duration was $2.9 \pm 1.1 \mathrm{mg}$ daily. Consistent with the overall population [27], $\mathrm{HbA}_{1 \mathrm{c}}$ over time did not differ between treatment groups in the CAROLINA-COGNITION study (ESM Fig. 4), although some initial differences were noted, e.g., adjusted mean $(95 \% \mathrm{CI})$ difference between linagliptin and glimepiride at week 16 and week 256: $2.84(2.37,3.32) \mathrm{mmol} / \mathrm{mol}(0.26$ 


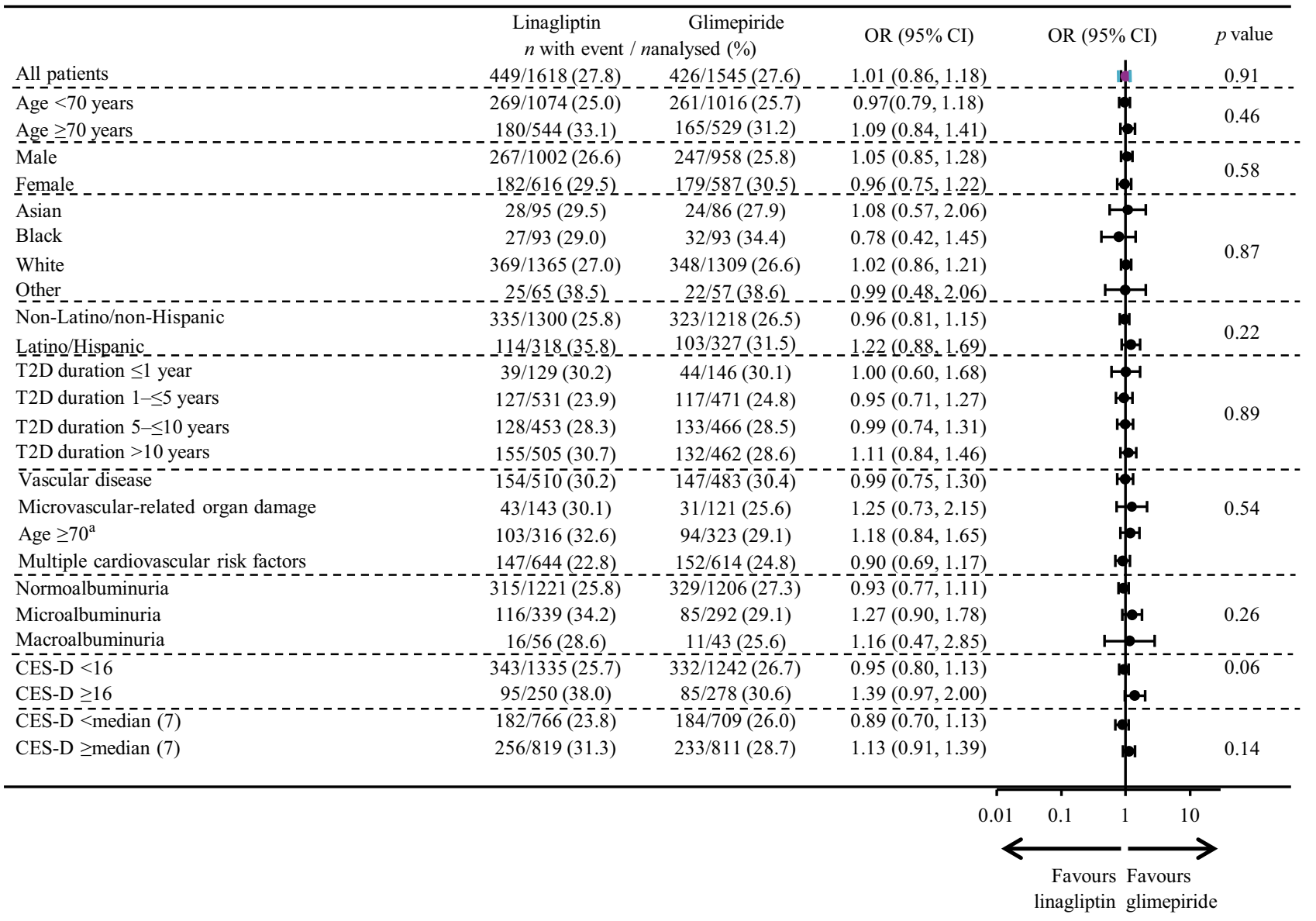

b

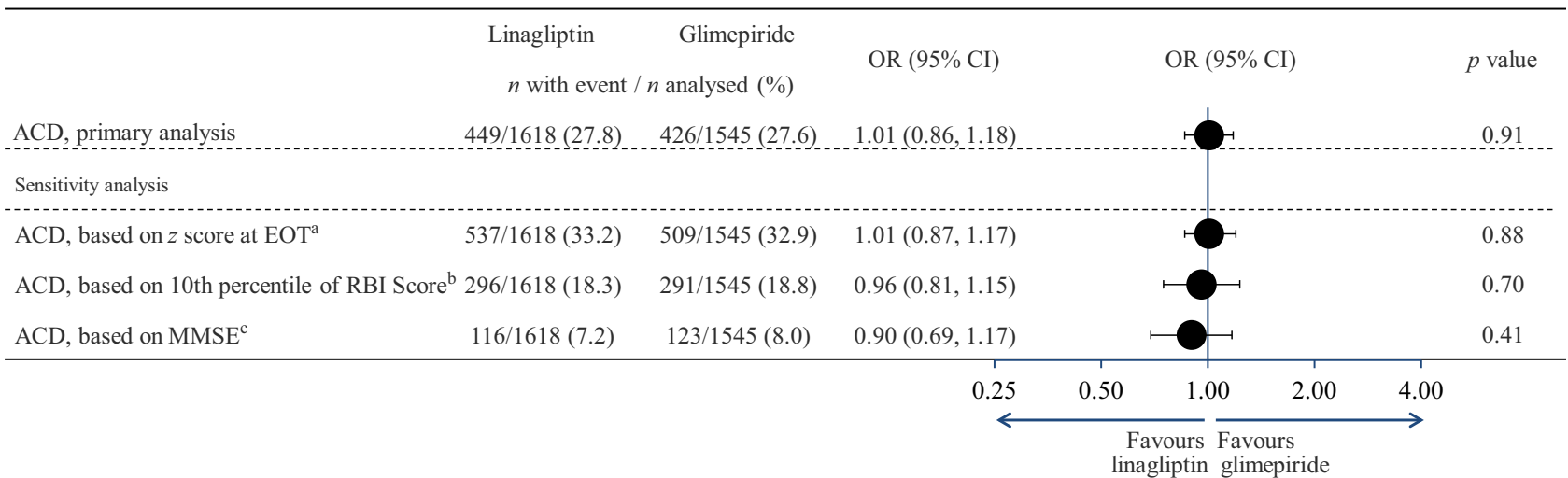

Fig. 1 Effect on ACD with linagliptin vs glimepiride at end of treatment. (a) Primary analysis and effects in prespecified subgroups. $p$ values depict overall treatment effect (top value) or treatment-by-subgroup interaction. ${ }^{a}$ The lower age-group relates to number of participants enrolled specifically according to the age-specific inclusion-criterion. (b) Secondary analysis of effects on ACD with linagliptin vs glimepiride. Treatment

$[0.22,0.30] \%)$, and $0.02(-0.96,1.00) \mathrm{mmol} / \mathrm{mol}(0.00$ $[-0.09,0.09] \%)$, respectively. Modest weight gain was observed in the glimepiride group early in the study and maintained thereafter, e.g., adjusted mean (95\% CI) difference between linagliptin and glimepiride at week 16 and week group values are $n$ with event $/ n$ analysed (\%). EOT, end of treatment, T2D, type 2 diabetes. ${ }^{a}$ Incidence of ACD based on 16th percentile of $z$ score for MMSE and/or attention and executive functioning. ${ }^{\mathrm{b}}$ Incidence of ACD based on 10th percentile of RBI score. 'Incidence of ACD at EOT based on MMSE score of $<24$ or a decline of $>4$ points in MMSE score at EOT relative to baseline

256: $-1.42(-1.61,-1.22) \mathrm{kg}$, and $-1.77(-2.22,-1.33) \mathrm{kg}$, respectively (ESM Fig. 5).

The incidence of hypoglycaemic events was lower with linagliptin across all hypoglycaemia categories in a post hoc analysis (ESM Fig. 6). Rates of symptomatic investigator- 
Table 2 Absolute changes in MMSE, attention and executive function, TMT, VFT and CES-D by treatment

\begin{tabular}{|c|c|c|c|c|}
\hline & \multicolumn{2}{|l|}{ Week $160^{\mathrm{a}}$} & \multicolumn{2}{|c|}{ End of follow-up ${ }^{b}$} \\
\hline & $\begin{array}{l}\text { Linagliptin } \\
(n=1618)\end{array}$ & $\begin{array}{l}\text { Glimepiride } \\
(n=1545)\end{array}$ & $\begin{array}{l}\text { Linagliptin } \\
(n=1618)\end{array}$ & $\begin{array}{l}\text { Glimepiride } \\
(n=1545)\end{array}$ \\
\hline \multicolumn{5}{|l|}{ MMSE score } \\
\hline Baseline & $28.5 \pm 1.7$ & $28.5 \pm 1.7$ & $28.5 \pm 1.7$ & $28.5 \pm 1.7$ \\
\hline Follow-up & $28.2 \pm 2.1$ & $28.3 \pm 2.2$ & $28.1 \pm 2.7$ & $28.1 \pm 2.7$ \\
\hline Change from baseline & $-0.2 \pm 2.0$ & $-0.2 \pm 2.1$ & $-0.4 \pm 2.6$ & $-0.4 \pm 2.6$ \\
\hline \multicolumn{5}{|l|}{$\mathrm{A} \& \mathrm{E}(z \text { score })^{\mathrm{c}}$} \\
\hline Baseline & $-0.01 \pm 0.70$ & $0.01 \pm 0.01$ & $-0.01 \pm 0.70$ & $0.01 \pm 0.01$ \\
\hline Follow-up & $-0.06 \pm 0.73$ & $-0.05 \pm 0.71$ & $-0.10 \pm 0.77$ & $-0.09 \pm 0.78$ \\
\hline Change from baseline & $-0.05 \pm 0.74$ & $-0.06 \pm 0.72$ & $-0.11 \pm 0.81$ & $-0.14 \pm 0.81$ \\
\hline \multicolumn{5}{|l|}{ VFT letter 60 (in seconds) ${ }^{\mathrm{d}}$} \\
\hline Baseline & $9.2 \pm 4.2$ & $9.3 \pm 4.1$ & $9.2 \pm 4.2$ & $9.3 \pm 4.1$ \\
\hline Follow-up & $8.9 \pm 4.0$ & $9.1 \pm 4.0$ & $9.1 \pm 4.3$ & $9.1 \pm 4.5$ \\
\hline Change from baseline & $-0.2 \pm 2.6$ & $-0.2 \pm 2.7$ & $-0.2 \pm 2.9$ & $-0.2 \pm 3.4$ \\
\hline \multicolumn{5}{|c|}{ VFT animals 60 (in seconds) ${ }^{\mathrm{e}}$} \\
\hline Baseline & $16.1 \pm 6.6$ & $16.0 \pm 6.2$ & $16.1 \pm 6.6$ & $16.0 \pm 6.2$ \\
\hline Follow-up & $15.6 \pm 6.6$ & $15.6 \pm 6.5$ & $15.7 \pm 7.5$ & $15.4 \pm 7.1$ \\
\hline Change from baseline & $-0.5 \pm 6.0$ & $-0.4 \pm 5.7$ & $-0.6 \pm 7.4$ & $-0.6 \pm 6.7$ \\
\hline \multicolumn{5}{|l|}{ Overall VFT 60 ( $z$ score $)$} \\
\hline Baseline & $-0.01 \pm 0.89$ & $0.002 \pm 0.85$ & $-0.01 \pm 0.89$ & $0.002 \pm 0.85$ \\
\hline Follow-up & $-0.09 \pm 0.84$ & $-0.07 \pm 0.84$ & $-0.06 \pm 0.96$ & $-0.08 \pm 0.96$ \\
\hline Change from baseline & $-0.07 \pm 0.63$ & $-0.06 \pm 0.64$ & $-0.07 \pm 0.78$ & $-0.09 \pm 0.76$ \\
\hline \multicolumn{5}{|l|}{ TMT-A (in seconds) } \\
\hline Baseline & $51.2 \pm 28.1$ & $50.4 \pm 26.7$ & $51.2 \pm 28.1$ & $50.4 \pm 26.7$ \\
\hline Follow-up & $53.7 \pm 30.3$ & $53.3 \pm 30.6$ & $54.1 \pm 30.6$ & $55.4 \pm 33.3$ \\
\hline Change from baseline & $2.1 \pm 24.2$ & $2.8 \pm 25.4$ & $3.4 \pm 25.6$ & $4.4 \pm 28.1$ \\
\hline \multicolumn{5}{|l|}{ TMT-B (in seconds) } \\
\hline Baseline & $113.1 \pm 57.8$ & $111.8 \pm 61.3$ & $113.1 \pm 57.8$ & $111.8 \pm 61.3$ \\
\hline Follow-up & $118.2 \pm 64.4$ & $118.3 \pm 63.8$ & $125.5 \pm 67.7$ & $125.3 \pm 66.0$ \\
\hline Change from baseline & $7.1 \pm 53.9$ & $9.8 \pm 54.1$ & $15.2 \pm 63.3$ & $18.2 \pm 59.5$ \\
\hline \multicolumn{5}{|l|}{ TMT ratio $^{f}$} \\
\hline Baseline & $1.4 \pm 1.0$ & $1.4 \pm 1.0$ & $1.4 \pm 1.0$ & $1.4 \pm 1.0$ \\
\hline Follow-up & $1.4 \pm 1.0$ & $1.4 \pm 1.0$ & $1.6 \pm 1.1$ & $1.5 \pm 1.0$ \\
\hline Change from baseline & $0.1 \pm 1.1$ & $0.2 \pm 1.1$ & $0.2 \pm 1.3$ & $0.3 \pm 1.0$ \\
\hline \multicolumn{5}{|l|}{ CES-D score } \\
\hline Baseline & $8.7 \pm 8.0$ & $9.3 \pm 8.3$ & $8.7 \pm 8.0$ & $9.3 \pm 8.3$ \\
\hline Follow-up & $10.5 \pm 9.9$ & $11.4 \pm 10.6$ & $10.2 \pm 9.9$ & $10.6 \pm 9.6$ \\
\hline Change from baseline & $1.7 \pm 9.6$ & $2.1 \pm 10.4$ & $1.6 \pm 9.6$ & $1.4 \pm 10.0$ \\
\hline
\end{tabular}

Data are mean \pm SD

Time between randomisation and visit: median (min-max): ${ }^{\mathrm{a}} 3.07(0.02-3.19),{ }^{\mathrm{b}} 6.40(3.20-7.42)$ years

${ }^{\mathrm{c}}$ A\&E, composite $z$ score for attention and executive functioning

${ }^{\mathrm{d}}$ VFT letter 60: averaged VFT scores for the letters F, A and S in $60 \mathrm{~s}$

${ }^{\mathrm{e}}$ VFT animals 60: VFT score for the category animals in $60 \mathrm{~s}$

${ }^{\mathrm{f}}$ TMT ratio: (TMT-B - TMT-A)/TMT-A

政

defined hypoglycaemia events with plasma glucose $\leq 3.9 \mathrm{mmol} / 1$, plasma glucose $<3.1 \mathrm{mmol} / 1$, or severe hypoglycaemic events were 1.30 events per 100 participant years for linagliptin and 8.61 per 100 participant years for glimepiride (HR 0.17 [95\% CI 0.14, 0.20]); rates of severe hypoglycaemic events were 0.07 and 0.48 per 100 participant 
years, respectively $(0.14[0.06,0.32])$. There were very few hospitalisations due to hypoglycaemic events (one event in the linagliptin group and 15 events in the glimepiride group; 0.01 events per 100 participant years for linagliptin and 0.17 per 100 participant years for glimepiride; $0.06[<0.01,0.48])$.

\section{Discussion}

In this comparative cognitive interventional trial of 3163 individuals with relatively early type 2 diabetes without impaired cognition at baseline, and elevated cardiovascular risk, the effects of linagliptin $5 \mathrm{mg}$ or glimepiride $1-4 \mathrm{mg}$ did not differ for the risk of ACD when assessed using three different instruments over a median follow-up of 6.12 years. This result was observed in the setting of glycaemic equipoise between treatment groups, but with differing effects on hypoglycaemia and weight.

These findings have biological implications as, in the context of neutral effects on ACD with linagliptin compared with placebo [26], they do not support previous hypotheses that DPP-4 inhibitors or sulfonylureas may affect cognitive function positively. However, the neutral effect on cognitive function also provides reassurance that these medications do not differentially affect cognitive performance, which has been debated for other glucose-lowering medications, e.g., metformin and insulin, albeit with conflicting data [14-17].

The duration of the substudy was driven by the number of cardiovascular events in the main study, and this led to a reasonably long observation period. As the progress of cognitive decline is usually slow, this is a strength of the present substudy. Another strength is the consistency using the more stringent 10th percentile as cut-off for ACD. With this cut-off we captured a group with substantial cognitive decline (i.e. $>3$ MMSE points on average). The data also show that in the group without ACD, according to our definitions, cognitive test performance was essentially stable over the course of 6 years, highlighting the importance of a dichotomous rather than continuous cognitive outcome measure for a trial if one intends to prevent clinically meaningful cognitive decline. Clearly a clinical diagnosis of dementia or MCI, based on a full diagnostic evaluation combined with elaborate cognitive testing and ideally adjudication, would have been even more meaningful as an outcome measure, however this is currently not feasible in a large international outcome trial such as CAROLINA, and therefore is a limitation of this substudy. Moreover, the CAROLINA-COGNITION study involved the MMSE, TMT and VFT as the cognitive testing battery, and it is uncertain if results would have looked different using other testing batteries or subjective cognitive complaints measures $[24,34]$.

The number of participants analysed both with global cognitive functioning and domain-specific function items was relatively large, and although the ADVANCE trial had a larger number of participants $(n=11,132)$, this was not a blinded placebo-controlled trial, it was designed to assess effects of intensive glucose lowering on cognitive impairment, and only used MMSE [8]. Prior to CAROLINACOGNITION, there had been very few RCTs that have been designed to investigate non-glycaemic effects of pharmacological interventions on cognition in type 2 diabetes in a direct comparative way, with most being too small or with too short duration to adequately address this question $[13,25]$. In CAROLINA, no difference in $\mathrm{HbA}_{1 \mathrm{c}}$ was observed. However, there was a marked difference in risk for hypoglycaemia across treatment arms, which is of relevance since hypoglycaemia, in particular severe events, has been correlated with cognitive impairment [23]. The role of hypoglycaemia in relation to the risk of MCI and dementia is, however, debated [24]. In the present substudy, there were very few episodes of severe hypoglycaemia, and too few to reliably analyse this relationship, and whether the results would have looked different in populations more susceptible to hypoglycaemia is unknown. Also, while greater weight in mid-life is associated with poorer cognitive function in individuals with type 2 diabetes [35], the cognitive function tests applied might not be sufficiently sensitive to the relatively modest magnitude of weight changes observed in this trial.

It should be noted that we observed a relatively large difference between numbers of participants with baseline assessment $(n=4529)$ and follow-up assessment $(n=3163)$. The main reason for this was due to protocol specification not to include cognitive assessments more than 7 days after end of treatment. In addition, several participants prematurely discontinued the study drug, at a rate also observed in other trials, and some died, which was part of the primary outcome of the trial. Nonetheless, rates were equally distributed across both treatment arms and the neutral result was reflected in subgroups and in sensitivity analyses, including using the broader participant dataset $(n=3452 ; 86 \%$ of those included at baseline). However, the generalisability of these results does not necessarily extend to those at higher risk for cognitive decline, or to those with MMSE $<24$, and we also cannot extrapolate the results to individuals with more advanced type 2 diabetes, or to other DPP-4 inhibitors or sulfonylureas.

An interesting exploratory observation from this substudy was that approximately a quarter of the population had shown signs of depressive symptoms at week 160 , and fewer in the linagliptin group (OR $0.84[0.71,1.00]$ ), a finding that was attenuated at end of follow-up (OR $0.86[0.71,1.05]$ ). Although both differing hypoglycaemia burden and weight gain could play a role [36], this substudy cannot provide definitive answers, and further studies are required.

In conclusion, in a population with relatively early type 2 diabetes, with intact cognitive function at baseline and at elevated risk for cardiovascular events, there was no 
difference in the rate of ACD between those treated with linagliptin or glimepiride. Additionally, this trial demonstrated that cognitive assessment in a large international outcome trial is feasible.

Supplementary Information The online version contains peer-reviewed but unedited supplementary material available at https://doi.org/10.1007/ s00125-021-05393-8.

Acknowledgements Parts of these data were accepted as an abstract for the EASD Annual Meeting, 2019. The authors thank the investigators, coordinators and patients who participated in this trial. We thank A. Passera, Biostatistics and Programming Department, HMS Analytical Software, Sulzbach, Germany (at the time of the conduct of this study), for her analytical contribution, funded by Boehringer Ingelheim, and S. Abu, Biostatistics and Programming Department, Mainanalytics $\mathrm{GmbH}$, Sulzbach, Germany, for analytical contribution of the revised manuscript, also funded by Boehringer Ingelheim.

Data availability The datasets generated during and/or analysed during the current study are available from the corresponding author on reasonable request.

Funding This study was sponsored by Boehringer Ingelheim. Employees of Boehringer Ingelheim were involved in the design and conduct of the study; collection, analysis, and interpretation of data; and preparation of this manuscript.

Authors' relationships and activities GJB has received research grants awarded to his institution from Boehringer Ingelheim. GW is an employee of Boehringer Ingelheim. BZ has received research grants awarded to his institution from Boehringer Ingelheim, AstraZeneca and NovoNordisk, honoraria from Janssen, Sanofi and Eli Lilly and Company, and honoraria from Boehringer Ingelheim, NovoNordisk and Merck. MAE has received honoraria from Boehringer Ingelheim. OEJ was an employee of Boehringer Ingelheim at the time of preparation of the manuscript, but is now employed by Nestlé Health Science. All other authors declare that there are no relationships or activities that might bias, or be perceived to bias, their contribution to this manuscript.

Contribution statement All authors fulfil the ICMJE criteria for authorship, are fully responsible for all content and editorial decisions, and were involved at all stages of manuscript development. GJB, CV and OEJ wrote the first draft of the manuscript, and OEJ drafted the revision. GJB, JJ, CV, GW and OEJ designed the statistical analyses. GW and $\mathrm{CV}$ carried out the statistical analyses. All authors have approved the final manuscript. GJB is the guarantor of this work.

Open Access This article is licensed under a Creative Commons Attribution 4.0 International License, which permits use, sharing, adaptation, distribution and reproduction in any medium or format, as long as you give appropriate credit to the original author(s) and the source, provide a link to the Creative Commons licence, and indicate if changes were made. The images or other third party material in this article are included in the article's Creative Commons licence, unless indicated otherwise in a credit line to the material. If material is not included in the article's Creative Commons licence and your intended use is not permitted by statutory regulation or exceeds the permitted use, you will need to obtain permission directly from the copyright holder. To view a copy of this licence, visit http://creativecommons.org/licenses/by/4.0/.

\section{References}

1. Biessels GJ, Despa F (2018) Cognitive decline and dementia in diabetes mellitus: mechanisms and clinical implications. Nat Rev Endocrinol 14:591-604. https://doi.org/10.1038/ s41574-018-0048-7

2. Stefanidis KB, Askew CD, Greaves K, Summers MJ (2018) The effect of non-stroke cardiovascular disease states on risk for cognitive decline and dementia: a systematic and meta-analytic review. Neuropsychol Rev 28:1-15. https://doi.org/10.1007/s11065-0179359-z

3. Biessels GJ, Strachan MW, Visseren FL, Kappelle LJ, Whitmer RA (2014) Dementia and cognitive decline in type 2 diabetes and prediabetic stages: towards targeted interventions. Lancet Diabetes Endocrinol 2:246-255. https://doi.org/10.1016/S2213-8587(13) 70088-3

4. Haroon NN, Austin PC, Shah BR, Wu J, Gill SS, Booth GL (2015) Risk of dementia in seniors with newly diagnosed diabetes: a population-based study. Diabetes Care 38:1868-1875. https://doi. org/10.2337/dc15-0491

5. Moore EM, Mander AG, Ames D et al (2013) Increased risk of cognitive impairment in patients with diabetes is associated with metformin. Diabetes Care 36(10):2981-2987. https://doi.org/10. 2337/dc13-0229

6. Exalto LG, Biessels GJ, Karter AJ et al (2013) Risk score for prediction of 10 year dementia risk in individuals with type 2 diabetes: a cohort study. Lancet Diabetes Endocrinol 1:183-190. https:// doi.org/10.1016/S2213-8587(13)70048-2

7. International Diabetes Federation (2019) IDF diabetes atlas, 9th edn. International Diabetes Federation, Brussels. Available from: https://www.diabetesatlas.org/en/resources/. Accessed Oct 132020

8. de Galan BE, Zoungas S, Chalmers J et al (2009) Cognitive function and risks of cardiovascular disease and hypoglycemia in patients with type 2 diabetes: the action in diabetes and vascular disease: Preterax and Diamicron Modified Release Controlled Evaluation (ADVANCE) trial. Diabetologia 52:2328-2336. https://doi.org/10.1007/s00125-009-1484-7

9. Groeneveld ON, van den Berg E, Johansen OE et al (2019) Oxidative stress and endothelial dysfunction are associated with reduced cognition in type 2 diabetes. Diab Vasc Dis Res 16:577581. https://doi.org/10.1177/1479164119848093

10. Verma N, Despa F (2019) Contributing factors to diabetic brain injury and cognitive decline. Diabetes Metab J 43:560-567. https://doi.org/10.4093/dmj.2019.0153

11. Crane PK, Walker R, Hubbard RA et al (2013) Glucose levels and risk of dementia. N Engl J Med 369:540-548. https://doi.org/10. 1056/NEJMoa1215740

12. Janssen J, van den Berg E, Zinman B et al (2019) $\mathrm{HbA}_{1 \mathrm{c}}$, insulin resistance and beta-cell function in relation to cognitive function in type 2 diabetes: the CAROLINA ${ }^{\circledR}$ cognition sub-study. Diabetes Care 42:e1-e3

13. Areosa Sastre A, Vernooij RW, González-Colaço Harmand M, Martínez G (2017) Effect of the treatment of type 2 diabetes mellitus on the development of cognitive impairment and dementia. Cochrane Database Syst Rev 6:CD003804. https://doi.org/10.1002/ 14651858.CD003804.pub2

14. Imfeld P, Bodmer M, Jick SS, Meier CR (2012) Metformin, other antidiabetic drugs, and risk of Alzheimer's disease: a population based case-control study. J Am Geriatr Soc 60:916-921. https:// doi.org/10.1111/j.1532-5415.2012.03916.x

15. Luschinger JA, Ma Y, Christophi CA et al (2017) Metformin, lifestyle intervention, and cognition in the diabetes prevention program outcomes study. Diabetes Care 40:958-965. https://doi.org/10. 2337/dc16-2376 
16. Weinstein G, Davis-Plourde KL, Conner S et al (2019) Association of metformin, sulfonylurea and insulin use with brain structure and function and risk of dementia and Alzheimer's disease: pooled analysis from 5 cohorts. PLoS One 14:e0212293. https://doi.org/ 10.1371/journal.pone.0212293

17. Cukierman-Yaffe T, Bosch J, Diaz R et al (2014) Effects of basal insulin glargine and omega-3 fatty acid on cognitive decline and probable cognitive impairment in people with dysglycaemia: a substudy of the ORIGIN trial. Lancet Diabetes Endocrinol 2:562572. https://doi.org/10.1016/S2213-8587(14)70062-2

18. Athauda D, Maclagan K, Skene SS et al (2017) Exenatide once weekly versus placebo in Parkinson's disease: a randomised, double-blind, placebo-controlled trial. Lancet 390:1664-1675. https://doi.org/10.1016/S0140-6736(17)31585-4

19. Shannon RP (2013) DPP-4 inhibition and neuroprotection: do mechanisms matter? Diabetes 62:1029-1031. https://doi.org/10. 2337/db12-1794

20. Cukierman-Yaffe T, Gerstein HC, Colhoun HM et al (2020) Effect of dulaglutide on cognitive impairment in type 2 diabetes: an exploratory analysis of the REWIND trial. Lancet Neurol 19:582590. https://doi.org/10.1016/S1474-4422(20)30173-3

21. Lin Z, Huang H, Gu Y et al (2017) Glibenclamide ameliorates cerebral edema and improves outcomes in a rat model of status epilepticus. Neuropharmacology 121:1-11. https://doi.org/10. 1016/j.neuropharm.2017.04.016

22. Hussien NR, Al-Naimi MS, Rasheed HA, Al-Kuraishy HM, AlGareeb AI (2018) Sulfonylurea and neuroprotection: the bright side of the moon. J Adv Pharm Technol Res 9:120-123. https://doi.org/ 10.4103/japtr.JAPTR $317 \quad 18$

23. Lee AK, Rawlings AM, Lee CJ et al (2018) Severe hypoglycemia, mild cognitive impairment, dementia and brain volumes in older adults with type 2 diabetes: the Atherosclerosis Risk in Communities (ARIC) cohort study. Diabetologia 61:1956-1965. https://doi.org/10.1007/s00125-018-4668-1

24. Cukierman-Yaffe T, Bosch J, Jung H, Punthakee Z, Gerstein HC (2019) Hypoglycemia and incident cognitive dysfunction: a posthoc analysis from the ORIGIN trial. Diabetes Care 42:142-147

25. Fink HA, Jutkowitz E, McCarten JR et al (2018) Pharmacologic interventions to prevent cognitive decline, mild cognitive impairment, and clinical Alzheimer-type dementia: a systematic review. Ann Intern Med 168:39-51. https://doi.org/10.7326/M17-1529

26. Biessels GJ, Verhagen C, Janssen J et al (2019) Effect of linagliptin on cognitive performance in patients with type 2 diabetes and Cardiorenal comorbidities: the CARMELINA randomized trial. Diabetes Care 42:1930-1938. https://doi.org/10.2337/dc19-0783
27. Rosenstock J, Kahn SE, Johansen OE et al (2019) Effect of linagliptin vs glimepiride on major adverse cardiovascular outcomes in patients with type 2 diabetes: the CAROLINA randomized clinical trial. JAMA 322:1155-1166. https://doi.org/ 10.1001/jama.2019.13772

28. Biessels GJ, Janssen J, van den Berg E et al (2018) Rationale and design of the CAROLINA ${ }^{\circledR}$ - cognition substudy: a randomised controlled trial on cognitive outcomes of linagliptin versus glimepiride in patients with type 2 diabetes mellitus. BMC Neurol 18:7

29. Folstein MF, Folstein SE, McHugh PR (1975) "Mini-mental state." A practical method for grading the cognitive state of patients for the clinician. J Psychiatr Res 12:189-198. https://doi.org/10.1016/ 0022-3956(75)90026-6

30. Tombaugh TN, McIntyre N (1992) The mini-mental state examination: a comprehensive review. J Am Geriatr Soc 40:922-935

31. Corrigan JD, Hinkeldey NS (1987) Relationships between parts A and B of the trail making test. J Clin Psychol 43:402-409. https:// doi.org/10.1002/1097-4679(198707)43:4<402::AIDJCLP2270430411>3.0.CO;2-E

32. Shao Z, Janse E, Visser K, Meyer AS (2014) What do verbal fluency tasks measure? Predictors of verbal fluency performance in older adults. Front Psychol 5:772. https://doi.org/10.3389/fpsyg.2014. 00772

33. Radloff LS (1977) The CES-D scale. A self-report depression scale for research in the general population. Appl Psychol Meas 1:385401

34. Jonker C, Geerlings MI, Schmand B (2000) Are memory complaints predictive for dementia? A review of clinical and population-based studies. Int J Geriatr Psychiatry 15:983-991. https://doi.org/10.1002/1099-1166(200011)15:11<983::AIDGPS238>3.0.CO;2-5

35. Espeland MA, Rapp SR, Bray GA et al (2014) Long-term impact of behavioral weight loss intervention on cognitive function: the Action for Health in Diabetes Movement and Memory study. J Gerontol A Biol Sci Med Sci 69:1101-1108. https://doi.org/10. 1093/gerona/glu031

36. Kikuchi Y, Iwase M, Fujii H et al (2015) Association of severe hypoglycemia with depressive symptoms in patients with type 2 diabetes: the Fukuoka Diabetes Registry. BMJ Open Diabetes Res Care 3:e000063. https://doi.org/10.1136/bmjdrc-2014-000063

Publisher's note Springer Nature remains neutral with regard to jurisdictional claims in published maps and institutional affiliations. 